\title{
Extent of eosinophilic esophagitis predicts response to treatment
}

\section{(ㄷ)(1)}

Authors

Hassan Ghoz ${ }^{1}$, Fernando F. Stancampiano ${ }^{2}$, Jose R. Valery ${ }^{2}$, Katie Nordelo ${ }^{3}$, Balkishan Malviya ${ }^{1}$, Brian E. Lacy ${ }^{1}$, Dawn Francis ${ }^{1}$, Kenneth DeVault ${ }^{1}$, Ernest Bouras ${ }^{1}$, Murli Krishna ${ }^{4}$, William C. Palmer ${ }^{1}$

Institutions

1 Division of Gastroenterology and Hepatology, Mayo Clinic, Jacksonville, Florida, United States

2 Division of Internal Medicine, Mayo Clinic, Jacksonville, Florida, United States

3 Clinical Research Internship Study Program (CRISP), Mayo Clinic, Jacksonville, Florida, United States

4 Department of Laboratory Medicine and Pathology, Mayo Clinic, Jacksonville, Florida, United States

submitted 18.11.2020

accepted after revision 15.3.2021

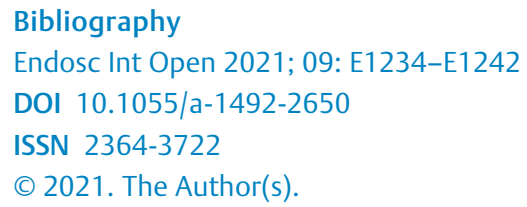
Commons Attribution-NonDerivative-NonCommercial License, permitting copying and reproduction so long as the original work is given appropriate credit. Contents may not be used for commercial purposes, or adapted, remixed, transformed or built upon. (https://creativecommons.org/licenses/by-nc-nd/4.0/)

Georg Thieme Verlag KG, Rüdigerstraße 14,

70469 Stuttgart, Germany

Corresponding author William C. Palmer, MD, Division of Gastroenterology and Hepatology, Mayo Clinic, 4500 San Pablo Road, Jacksonville, FL 32224, United States

Palmer.william@mayo.edu

Supplementary material is available under https://doi.org/10.1055/a-1492-2650
ABSTRACT

Background and study aim The clinical impact of eosinophilic esophagitis (EoE) limited to the distal esophagus (Lim-EE) vs. diffuse involvement (Dif-EE) is unknown. This study compared clinical characteristics and outcomes of Lim-EE vs. Dif-EE.

Patients and methods This retrospective, single-center study of patients with EoE between December 2011 and December 2019 evaluated treatment response based on repeated pathology and/or clinical improvement using comparative statistics.

Results 479 patients were identified (126 Lim-EE, 353 Dif-EE). Lim-EE patients had a higher incidence of endoscopically identified esophagitis $(23.0 \%$ vs. $14.7 \%$; $P=$ 0.04 ), were older (50.8 [SD 16.2] vs. 46.4 [SD 15.3] years; $P=0.007)$, and were more likely to present with iron deficiency anemia (5.6\% vs. $1.7 \% ; P=0.05)$, dyspepsia $(15.1 \%$ vs. $8.8 \% ; P=0.06)$ or for Barrett's surveillance $(10.3 \%$ vs. $3.7 \% ; P=0.02)$. Patients with Dif-EE presented more frequently with dysphagia (57.2\% vs. $45.2 \%$; $P=0.02)$. Both groups had similar proton pump inhibitor $(87.2 \%$ vs. $83.3 \% ; P=0.37)$ and steroid $(12.8 \%$ vs. $21.4 \% ; P=0.14)$ use. Patients with Lim-EE had a better clinicopathologic response ( $61.5 \%$ vs. $44.8 \%$; $P=0.009)$. On multivariate analysis, EoE extent predicted treatment response with an odds ratio of 1.89 ( $95 \%$ confidence interval 1.13-3.20; $P=0.02$ ). However, treatment response based only on repeat biopsy results showed no statistical difference between Lim-EE (52.5\%) and Dif-EE (39.7\%; $P=0.15)$.

Conclusions Lim-EE may represent a distinct phenotype separate from Dif-EE, with more overlap with gastroesophageal reflux disease and better treatment response.

\section{Introduction}

Eosinophilic esophagitis (EoE) is a leading cause of dysphagia and food impaction in young adults and a common cause of chronic esophagitis [1]. EoE is a clinicopathologic disorder defined both by symptoms and objective findings of eosinophilia (>15 eosinophils per high power field [hpf]) on esophageal biopsies [2]. Current guidelines recommend that at least six esophageal biopsies be obtained from two different locations in the esophagus, most typically the distal and proximal halves [3]. As the number of biopsies reaches six or more, the diagnostic sensitivity for EoE significantly improves [3,4]. Obtaining biopsies from different esophageal locations may also improve detection of EoE given its patchy nature [5]. 
Many endoscopists have adopted the practice of routinely separating esophageal biopsies into different jars based on their location (proximal vs. distal). This approach, although more expensive and somewhat more time-consuming, has been theorized by physicians to differentiate EoE from gastroesophageal reflux disease (GERD), with the assumption that eosinophils will be more numerous in the distal esophagus in GERD and more diffuse in EoE $[3,6]$. Some studies have supported this theory by showing significant proximal eosinophilia in adults diagnosed with EoE compared with GERD [7], while others have not $[8,9]$. While acknowledging the associated additional costs and the added procedural time and risks, the clinical benefits of separating pathology specimens into different jars remain unclear. Updated guidelines in 2011 raised the question of whether the distribution of inflammatory changes in EoE predicts response to proton pump inhibitor (PPI) therapy [10]. The current study was designed to address several of these unanswered questions. We hypothesized that biopsies from different locations could be important in assessing the extent and degree of inflammation in the esophagus. Biopsies from different locations could also help assess the degree of overlap with GERD. Finally, results of esophageal biopsies from distal and more proximal sites could be used to predict treatment response. The patchy nature of EoE may be a reflection of the extent of disease, while dense eosinophilia limited to the distal third of the esophagus may represent a milder form of EoE or an overlap with GERD that has better treatment response when compared with diffuse disease. Therefore, our study aimed to compare patient demographics, symptoms, clinical characteristics, and treatment outcomes of patients with limited EoE (Lim-EE) vs. those with diffuse EoE extending to the proximal parts of the esophagus (Dif-EE).

\section{Methods}

\section{Patient selection and study design}

This study was a retrospective review of patients who underwent esophagogastroduodenoscopy (EGD) with esophageal biopsies positive for eosinophils at Mayo Clinic in Jacksonville, Florida, between December 2011 and December 2019. Only patients who had at least one esophageal biopsy showing more than 15 eosinophils/hpf, obtained from both the distal and proximal esophagus and separated into different pathology specimen jars, were included. An average of 4 passes (2 biopsy pieces/pass) per patient were obtained. EoE was defined as the presence of $\geq 15$ eosinophils/hpf. Patients were subclassified into distal (Lim-EE) and diffuse (Dif-EE) based on the extent of EoE. The Lim-EE group was defined by localized eosinophilic involvement of the distal third of the esophagus and lack of eosinophilia in the proximal esophagus. The Dif-EE group was defined by esophageal eosinophilia in both the proximal and distal esophagus. We assessed treatment outcomes based on histopathologic and clinicopathologic response (see below). A summary of patient selection and study design is presented in - Fig. 1.
Patients with EGD/biopsies showing eosinophils $>15 \operatorname{HPF}(n=508)$

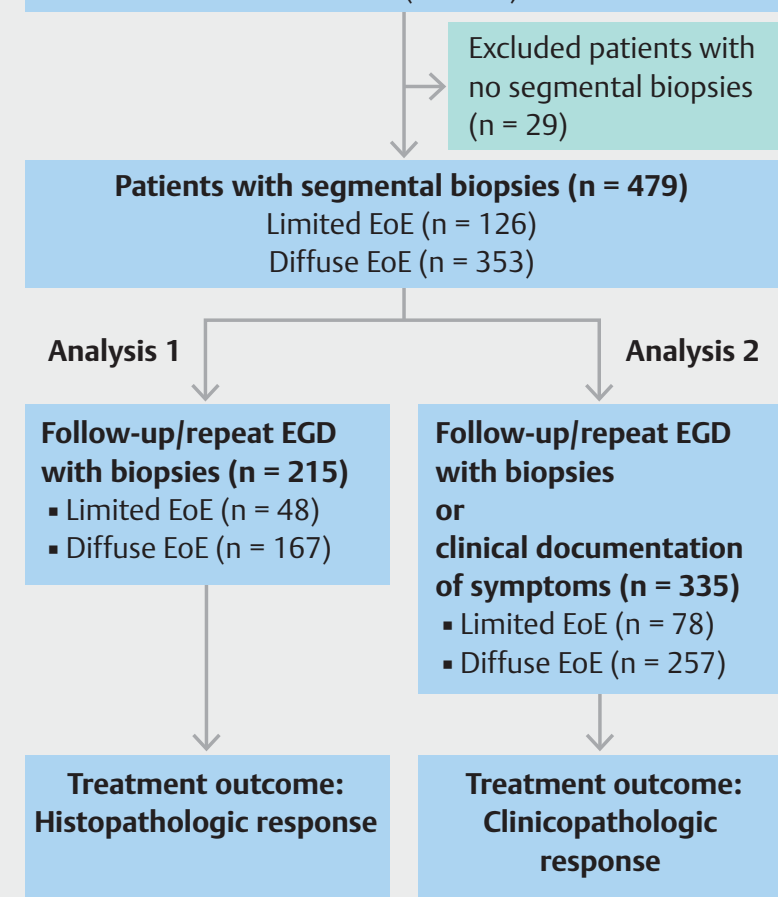

- Fig. 1 Summary of the patient selection and study design. EGD, esophagogastroduodenoscopy; HPF, high power field; EoE, eosinophilic esophagitis.

\section{Indications and protocol for obtaining segmental esophageal biopsies}

In our institution, we frequently perform segmental esophageal biopsies in patients who present with dysphagia, patients who have incidental endoscopic findings concerning for EoE, and patients who present for Barrett's surveillance in order to rule out dysplasia. Our protocol for obtaining segmental esophageal biopsies in patients with dysphagia or those who have endoscopic findings suggesting EoE is to obtain at least 2 passes (2 biopsy pieces/pass) from the lower distal third as well as the upper and/or middle third. Some endoscopists separate these into two jars, which was an inclusion criterion in our study, while others place them all in one jar. For Barrett's surveillance, it is common in our practice to obtain biopsies from the distal as well as middle thirds of the esophagus to rule out dysplasia. Indications for esophageal biopsies are summarized in $\mathbf{r}$ Table 1.

\section{Demographics and clinical characteristics}

Lim-EE and Dif-EE groups were compared based on patient demographics (age, sex, race, body mass index), relevant clinical characteristics (history of asthma, eczema, seasonal allergies), clinical presentation/indications, and findings on their initial diagnostic EGD including reflux esophagitis graded using the Los Angeles (LA) classification system, pertinent medications at the time of biopsies (e. g. PPI, steroids), relevant clinical testing for GERD if available (Bravo [Medtronic, Minneapolis, Minnesota, 
USA] and ambulatory $\mathrm{pH}$ monitoring test results), and disease complications such as food impaction and esophageal perforation. To determine the number of patients who were on PPI by the time of the initial or follow-up EGD, we manually checked the medication list for each patient on the day of the EGD.

\section{Comparing histopathologic treatment response}

This analysis involved patients who had a follow-up EGD with biopsies following their initial EGD. Response to treatment was defined as the presence of $<15$ eosinophils/hpf on both proximal and distal esophageal biopsies. The follow-up period was calculated as the time between initial and repeat endoscopies. We reviewed and collected treatment data at the time of both endoscopies including medical therapy (type and dose), food elimination diets, and esophageal dilations.

\section{Comparing clinicopathologic treatment response}

Patients included in this analysis were followed in one of two ways: they either underwent repeat EGD with biopsies or were followed clinically based on their symptoms. A positive treatment response was defined as physician-documented symptomatic improvement on follow-up or a histopathologic improvement ( $<15$ eosinophils/hpf) at the time of follow-up EGD. Symptomatic improvement was defined as resolution of the presenting symptom documented in physicians' notes. The follow-up period was calculated as the time between the initial EGD and repeat endoscopy or date of the clinical follow-up visit.

\section{Statistical analysis}

Continuous variables were summarized with the sample median and range. Categorical variables were summarized with number and percentage of patients. Comparisons between groups were made using a Wilcoxon rank sum test or Fisher's exact test. Univariate and multivariable logistic regression models were used to identify predictors for treatment response. All tests were two-sided with alpha level set at 0.05 for statistical significance. Statistical analyses were performed using JMP, Version 14.1.0 (SAS Institute Inc., Cary, North Carolina, USA, 1989-2019).

Institutional Review Board approval was obtained. Patients were not compensated in any form for participating in the study.

\section{Results}

\section{Patient demographics and clinical characteristic}

We initially included 508 patients who had an EGD with biopsies that showed >15 eosinophils/hpf between December 2011 and December 2019; however, we eventually excluded 29 patients who did not have segmental biopsies (i.e. biopsies were not taken from two separate locations in the esophagus and were not placed in different jars). Patients were grouped based on the location of esophageal eosinophilia. A total of 126 patients had Lim-EE and 353 had Dif-EE. A summary of patient demographics and clinical characteristic is shown in $>$ Table 1.

When compared with patients with Dif-EE, those with Lim-EE were older (mean 50.8 [SD 16.2] vs. 46.4 [SD 15.3] years; $P=$
$0.007)$ and had a higher incidence of endoscopically identified reflux esophagitis ( $23.0 \%$ vs. $14.7 \% ; P=0.04$ ), especially LA grade A esophagitis ( $9.5 \%$ vs. $3.4 \%$; $P=0.01$ ). Patients with Lim-EE were more likely to present with iron deficiency anemia (5.6\% vs. $1.7 \%$; $P=0.05)$, dyspepsia ( $15.1 \%$ vs. $8.8 \% ; P=0.06)$, or as part of a Barrett's surveillance program (10.3\% vs. $3.7 \%$; $P=0.02)$. In contrast, patients with Dif-EE presented more frequently with dysphagia vs. patients with Lim-EE $(57.2 \%$ vs. $45.2 \% ; P=0.02$ ). Only a small number of patients had Bravo testing and $\mathrm{pH}$ monitoring, with no statistically significant differences between the two groups.

\section{Treatment outcomes}

We compared the clinicopathologic treatment response of 78 patients with Lim-EE and 257 patients with Dif-EE. These were the subset of patients who underwent repeat EGD or had a follow-up clinic visit following their initial EGD. There were no differences in demographics or clinical characteristics between the two groups (Supplemental Table 1s). Both groups had similar treatment data (PPI use, dose and type, steroid use and type, elimination diet), as shown in $>$ Table 2.

Over $60 \%$ of patients $(61.5 \%)$ with Lim-EE $(n=48 / 78)$ responded to treatment based on repeated biopsy or documented clinical improvement, compared with $44.8 \%(n=115 / 257)$ in the Dif-EE group $(P=0.009)$ at a mean follow-up of 14.4 (SD 16.5) and 15.0 (SD 1.1) months $(P=0.81)$, respectively ( $>$ Fig.2). Based on improvement in clinical symptoms alone, $76.7 \%$ of patients with Lim-EE $(n=23 / 30)$ responded to treatment compared with $51.1 \%(n=46 / 90)$ in the Dif-EE group $(P=0.02)$.

We compared treatment response of 48 patients with Lim-EE and 167 patients with Dif-EE exclusively based on histopathology results of repeated esophageal biopsies. Indications for repeated EGD with biopsies are outlined in Supplementary Table 2s. Lim-EE patients were older (52.8 [SD 14.8] vs. 47.7 [SD 14.9]; $P=0.036)$ and had a higher incidence of endoscopically identified esophagitis on the initial EGD (27.1\% vs. $12.0 \%$; $P=$ 0.02 ), particularly grade $D$ esophagitis ( $8.3 \%$ vs. $1.8 \% ; P=$ 0.046) compared with Dif-EE (Supplemental Table 2s). Both groups received similar treatments including PPI dose and type, steroids dose and type, esophageal dilation, and elimination diet trial, as shown in - Table 3 . They also had similar follow-up time for repeated biopsies (12.5 [SD 16.8] and 14.1 [SD 18.2] months, respectively; $P=0.63$ ).

Assessing outcomes, Lim EE patients had a $22.9 \%$ reduction in reflux esophagitis observed at follow-up EGD $(P=0.002)$ compared with $4.8 \%$ in the Dif-EE group $(P=0.06)$. Histopathologic response was achieved in $52.5 \%$ of patients in the Lim-EE group and $39.7 \%$ in the Dif-EE group $(P=0.15)$. Results are illustrated in $\mathbf{F i g} .2$.

After adjusting for age and sex, the extent of esophageal involvement in EoE (Lim-EE vs. Dif-EE) predicted clinicopathologic treatment response with an adjusted odds ratio (aOR) of 1.89 (95\% confidence interval $[\mathrm{Cl}] 1.13-3.20 ; P=0.02$ ) and clinical response with an aOR of $3.12(95 \% \mathrm{Cl} 1.22-8.02 ; P=0.02)$. However, extent of esophageal involvement did not predict histologic response, with an aOR of $1.40(95 \% \mathrm{Cl} 1.40-0.31 ; P=$ $0.31)$. 
- Table 1 Demographics and clinical characteristics.

\begin{tabular}{|c|c|c|c|}
\hline & $\begin{array}{l}\text { Lim-EE } 1 \\
(n=126)\end{array}$ & $\begin{array}{l}\text { Dif-EE } 2 \\
(n=353)\end{array}$ & $P$ value \\
\hline Age, mean (SD), years & $50.8(16.2)$ & $46.4(15.3)$ & $0.007^{3}$ \\
\hline Sex (male), n (\%) & $77(61.1)$ & $216(61.2)$ & 0.55 \\
\hline Race (white), n (\%) & $118(93.7)$ & $335(94.9)$ & 0.65 \\
\hline BMI, mean (SD), kg/m² & $27.4(5.30)$ & $28.1(9.77)$ & 0.61 \\
\hline \multicolumn{4}{|c|}{ Indication for performing EGD with segmental biopsies, $\mathrm{n}(\%)$} \\
\hline - Dysphagia & $57(45.2)$ & $202(57.2)$ & $0.02^{3}$ \\
\hline \multicolumn{4}{|c|}{ - Incidental endoscopic EoE findings during evaluation for: } \\
\hline - GERD & $28(22.2)$ & $66(18.7)$ & 0.43 \\
\hline - Iron deficiency anemia & $7(5.6)$ & $6(1.7)$ & 0.05 \\
\hline - Dyspepsia & $19(15.1)$ & $31(8.8)$ & 0.06 \\
\hline - Food impaction & $3(2.4)$ & $8(2.3)$ & $>0.99$ \\
\hline - Other & $9(7.1)$ & $42(11.9)$ & - \\
\hline - Barrett's surveillance & $13(10.3)$ & $13(3.7)$ & $0.02^{3}$ \\
\hline History of asthma, n (\%) & $28(22.2)$ & $101(28.6)$ & 0.19 \\
\hline History of eczema, n (\%) & $12(9.5)$ & $26(7.4)$ & 0.46 \\
\hline History of seasonal allergies, n (\%) & $51(40.5)$ & $162(45.9)$ & 0.29 \\
\hline History of food impaction, n (\%) & $13(10.3)$ & $44(12.5)$ & 0.63 \\
\hline History of esophageal perforation, n (\%) & 0 & $4(1.1)$ & 0.58 \\
\hline Reflux esophagitis on EGD, ${ }^{4} \mathrm{n}(\%)$ & $29(23.0)$ & $52(14.7)$ & $0.04^{3}$ \\
\hline - LA A & $12(9.5)$ & $12(3.4)$ & $0.01^{3}$ \\
\hline . LAB & $9(7.1)$ & $24(6.8)$ & $>0.99$ \\
\hline - LAC & $4(3.2)$ & $10(2.8)$ & 0.77 \\
\hline - LAD & $4(3.2)$ & $6(1.7)$ & 0.29 \\
\hline PPI use before initial EGD/biopsy, n (\%) & $45(35.7)$ & $132(37.4)$ & 0.82 \\
\hline Esophageal dilation at time of biopsy, n (\%) & $30(23.8)$ & $84(23.8)$ & $>0.99$ \\
\hline Bravo ${ }^{5}$ testing, $\mathrm{n}(\%)$ & 12 & 23 & 0.40 \\
\hline - Positive test & $5(41.7)$ & $13(56.5)$ & \\
\hline Ambulatory $\mathrm{pH}$ monitoring, $\mathrm{n}(\%)$ & 9 & 15 & 0.39 \\
\hline - Positive test & $2(22.2)$ & $7(46.7)$ & \\
\hline $\begin{array}{l}\text { SD, standard deviation; BMI, body mass index; EC } \\
\text { ton pump inhibitor. } \\
{ }^{1} \text { EoE limited to the distal esophagus. } \\
{ }^{2} \text { EoE with diffuse involvement. } \\
{ }^{3} \text { Statistically significant. } \\
{ }^{4} \text { Los Angeles classification. } \\
{ }^{5} \text { Bravo pH Monitoring System (Medtronic, Minne }\end{array}$ & ; EoE, eosinophi & D, gastroesopha & ase; PPI, pr \\
\hline
\end{tabular}

We repeated our analysis to include patients with esophageal symptoms only (dysphagia, chest pain, dyspepsia, food impaction, and heartburn) and observed similar outcomes. Clinical improvement was documented in $60.5 \%$ (46/76) of the LimEE group compared with $44.8 \%(108 / 241)$ in the Dif-EE group $(P=0.02)$, while histologic response was observed in $51.2 \%$
$(21 / 41)$ in the Lim-EE group compared with $41.2 \%(63 / 153)$ in the Dif-EE group $(P=0.29)$.

For the clinicopathologic response, the number of patients who were on PPIs before the initial EGD was 30/78 (38.5\%) with Lim-EE and 101/257 (39.3\%) with Dif-EE. The number of patients who were on PPIs after the initial EGD (until the fol- 
- Table 2 Treatment data for patients included in the clinicopathologic response analysis.

\begin{tabular}{|c|c|c|c|c|c|c|c|}
\hline & $\begin{array}{l}\text { Lim-EE } \\
(n=78)\end{array}$ & $\begin{array}{l}\text { Dif-EE }{ }^{2} \\
(n=257)\end{array}$ & $P$ value & & $\begin{array}{l}\text { Lim-EE } 1 \\
(n=78)\end{array}$ & $\begin{array}{l}\text { Dif-EE } 2 \\
(n=257)\end{array}$ & $P$ value \\
\hline $\begin{array}{l}\text { Elimination diet before } \\
\text { initial EGD/biopsy, } \mathrm{n}(\%)\end{array}$ & 0 & $5(1.9)$ & 0.59 & $\begin{array}{l}\text { Elimination diet after initial } \\
\text { EGD/biopsy, } n(\%)\end{array}$ & $10(12.8)$ & $44(17.1)$ & 0.38 \\
\hline $\begin{array}{l}\text { PPI use before initial EGD/ } \\
\text { biopsy, n (\%) }\end{array}$ & $30(38.5)$ & $101(39.3)$ & 0.37 & $\begin{array}{l}\text { PPI use after initial EGD/ } \\
\text { biopsy, n (\%) }\end{array}$ & $68(87.2)$ & $214(83.3)$ & 0.37 \\
\hline - PPI BID dosing, n (\%) & $10(33.3)$ & $34(33.7)$ & 0.57 & - PPI BID dosing, n (\%) & $44(64.7)$ & $149(69.6)$ & 0.64 \\
\hline $\begin{array}{l}\text { - PPI total dose, mean } \\
\text { (SD), mg }\end{array}$ & $33.8(11.6)$ & $32.0(10.9)$ & 0.46 & $\begin{array}{l}\text { - PPI total dose, mean } \\
\text { (SD), mg, n (\%) }\end{array}$ & $55.1(20.3)$ & $56.3(22.6)$ & 0.68 \\
\hline \multicolumn{3}{|l|}{ PPI type, n } & & \multicolumn{3}{|l|}{ PPI type, n } & \\
\hline - Omeprazole & 33 & 117 & & - Omeprazole & 35 & 117 & \\
\hline - Esomeprazole & 8 & 27 & & - Esomeprazole & 8 & 21 & \\
\hline - Pantoprazole & 20 & 45 & & - Pantoprazole & 20 & 45 & \\
\hline - Dexlansoprazole & 0 & 2 & & - Dexlansoprazole & 0 & 2 & \\
\hline - Lansoprazole & 4 & 20 & & - Lansoprazole & 4 & 20 & \\
\hline - Rabeprazole & 1 & 1 & & - Rabeprazole & 1 & 2 & \\
\hline $\begin{array}{l}\text { Steroid use before initial } \\
\text { EGD/biopsy }\end{array}$ & $5(6.4)$ & $13(5.1)$ & 0.57 & $\begin{array}{l}\text { Steroid use after initial } \\
\text { EGD/biopsy }\end{array}$ & $10(12.8)$ & $55(21.4)$ & 0.14 \\
\hline - Fluticasone & 3 & 9 & & Fluticasone & 9 & 41 & \\
\hline - Budesonide & 2 & 4 & & Budesonide & 1 & 14 & \\
\hline
\end{tabular}

low-up date) was $68 / 78$ (87.2\%) with Lim-EoE and 214/257 $(83.3 \%)$ with Dif-EE, as shown in $>$ Table 2 . For histologic response, the number of patients who were on PPI before the initial EGD was 18/48 (37.5\%) with Lim-EE and 58/167 (34.7\%) with Dif-EE. The number of patients who were on PPIs after the initial EGD (until follow-up EGD) was 39/48 (81.3\%) with Lim-EE and $128 / 167$ (76.7\%) with Dif-EE.

We performed a subanalysis of the clinicopathologic response after excluding patients who where on PPIs or steroids at the time of biopsy during their initial EGD. The total number of patients excluded was 131 patients, leaving 48 patients in the Lim-EE group and 156 in the Dif-EE group to be included in this analysis. A total of 28/48 patients (58.3\%) with Lim-EE and $68 / 156$ (43.6\%) with Dif-EE had clinicopathologic response to treatment $(P=0.06)$. For histologic response, the total number of patients excluded from this analysis was 76 patients. After analysis, 41/109 patients (37.6\%) with Dif-EE responded histologically to treatment compared with $14 / 30$ (46.7\%) with Lim-EE $(P=0.52)$.

\section{Discussion}

EoE is an inflammatory disorder characterized by patches of eosinophilic mucosal infiltration involving single or multiple locations of the esophagus [11]. Current guidelines call for multiple biopsies from the proximal and distal esophagus to increase the sensitivity of the endoscopy [4]. Separating biopsies into different jars is a common practice by endoscopists; however, as this approach may result in significant monetary expense to the patient, is time-consuming, and confers some added risk, a better understanding of its clinical utility is warranted. Updated consensus statements have questioned the clinical value of defining the extent of EoE disease and the ability of this measure to predict treatment response $[3,10,12]$. In the current study, we found potential benefits of segmental biopsies; specifically, the extent of esophageal involvement may predict treatment response, as patients with EoE limited to the distal esophagus had a better clinicopathologic response compared with those with diffuse EoE.

Defining EoE and its correlation with GERD has been an area of active scientific inquiry over the past decade [13]. Early studies suggested a strong overlap between the two disease states $[14,15]$. Subsequent investigations led to a change in classification, such that these two disorders were thought to be distinctly separate based on response to PPI therapy $[12,16]$. Therefore, the current notion is that EoE and GERD share a mutually complex relationship and may indeed coexist. Although uncommon, EoE may cause reflux due to decreased esophageal compliance or alterations to normal esophageal motility, whereas GERD may disrupt the epithelial barrier integrity allowing antigen exposure and the eventual development of eosinophilia [17]. 


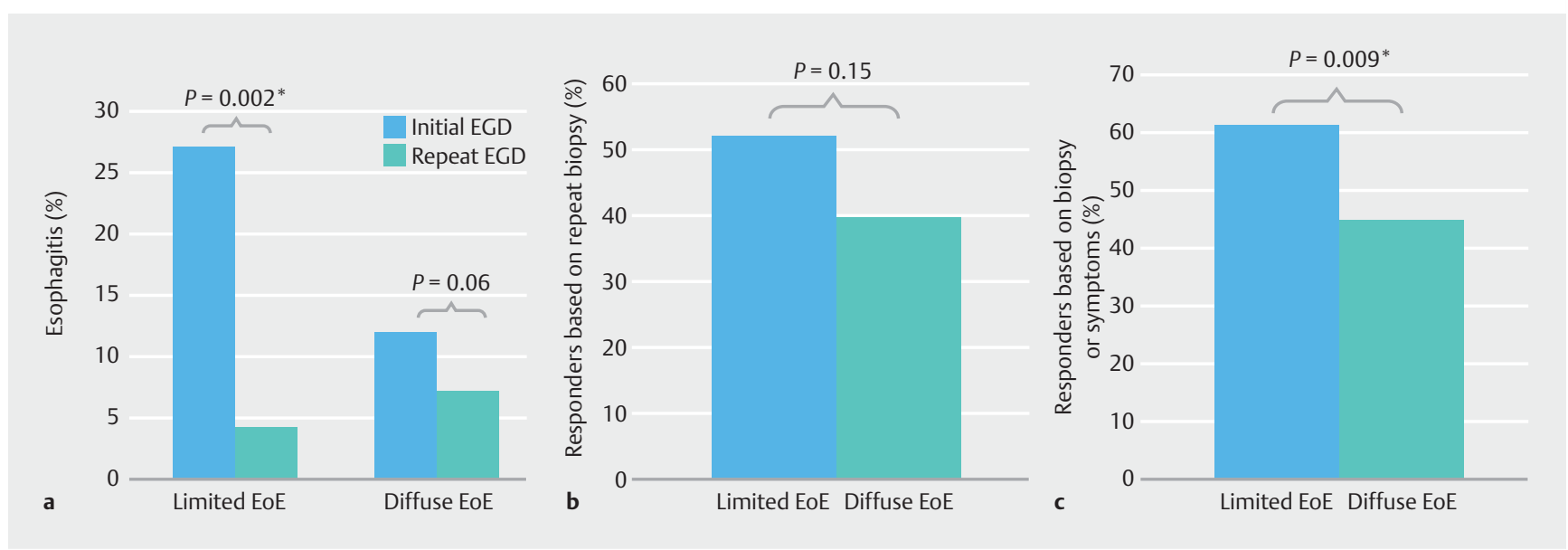

- Fig. 2 Response to treatment. a Observed esophagitis during repeat esophagogastroduodenoscopy. b Repeat biopsy results. c Repeat biopsy or clinical symptoms. EGD, esophagogastroduodenoscopy; EoE, eosinophilic esophagitis. *Statistically significant.

In our study, we hypothesized that patients with eosinophilia limited to the distal esophagus represent a population with GERD or mild EoE. A prior prospective study by Frazzoni et al. concluded that reflux plays a role in the pathogenesis of EoE, particularly in PPI-responsive cases [13]. In our cohort, Lim-EE patients were older, had a higher incidence of endoscopically identified reflux esophagitis, and presented with dyspepsia or as part of a Barrett's surveillance program; patients with Dif-EE had a higher incidence of dysphagia. We hypothesized that dysphagia is more common in Dif-EE due to the diffuse nature or changes in compliance and/or motility as this entity likely represents patients with EoE, compared with limited distal disease, which may be more compliant and have normal motility and likely corresponds to GERD or mild EoE.

Managing and evaluating treatment outcomes in EoE may be challenging due to the discordance between symptoms and histology [18]. The ultimate goal of treating EoE is to eliminate both patient symptoms as well as the eosinophilia ( $\geq 15$ eosinophils/hpf in biopsies). Treatment options may be delivered as monotherapy or combination of PPI therapy, topical steroids, elimination diet, and esophageal dilation [19]. In 2016, a meta-analysis that combined adult and pediatric patients reported PPI-induced clinical and histologic remission in $60.8 \%$ and $50.5 \%$ of the patients, respectively [20]. A follow-up study showed a $33 \%$ sustained clinical remission and histologic remission with $<15$ eosinophils/hpf on PPI therapy alone [21]. In addition, Lucendo et al. reported $58 \%$ complete remission (clinical and histologic) with oral budesonide tablets ( $1 \mathrm{mg}$ twice daily) compared with placebo [22].

In our study, we assessed treatment outcomes based on either histologic response alone or histologic and/or clinical response. Both Lim-EE and Dif-EE patients were treated similarly with about $80 \%$ of both groups receiving PPI therapy after their initial EGD. This may simply reflect the common clinical practice of treating EoE patients with a "step-up" approach that starts with PPI therapy followed by steroids. The Lim-EE group had a $22.9 \%$ reduction in esophagitis at follow-up EGD ( $P=$ 0.002 ) compared with $4.8 \%$ in the Dif-EE group $(P=0.06)$. We realize that reported esophagitis may have been secondary to EoE, although unlikely, or reflux, but given the lack of $\mathrm{pH}$ monitoring data, it was not possible to make such a distinction. However, in both scenarios, better mucosal healing and response to treatment in Lim-EE compared with Dif-EE may be considered. Furthermore, $61.5 \%$ of patients with Lim-EE achieved clinicopathologic response compared with $44.8 \%$ in the Dif-EE group $(P=0.009)$ at a similar follow-up period of about a year. Given the significant age difference between the groups, we performed a multivariate analysis adjusting for age and sex. In support of our hypothesis, disease extent predicted clinicopathologic response to treatment with an aOR of 1.89 (95\% Cl 1.13-3.20; $P=0.02)$. To better characterize the observed effect, we performed a subanalysis of treatment response based on improvement in clinical symptoms only and histopathologic response only. Clinically, $76.7 \%$ of patients with Lim-EE $(n=23 / 30)$ responded to treatment compared with $51.1 \%(n=46 / 90)$ in the Dif-EE group $(P=0.02)$. Numerical analysis of the histopathologic response to treatment showed a nonsignificant statistical difference in favor of the Lim-EE group (52.5\% vs. $39.7 \% ; P=0.15$ ). After adjusting for age and sex, the extent of EoE predicted treatment response based on clinical improvement with an aOR of 3.12 (95\%Cl 1.22-8.02; $P=0.02$ ); however, it did not predict histologic response, with an aOR of $1.40(95 \% \mathrm{Cl} 1.40-0.31 ; P=0.31)$. We repeated our analysis to include patients with esophageal symptoms only (dysphagia, chest pain, dyspepsia, food impaction, and heart burn) resulting in similar outcomes. Clinical improvement was documented in $60.5 \%(46 / 76)$ of the Lim-EE group compared with $44.8 \%$ $(108 / 241)$ in the Dif-EE group $(P=0.02)$, while histologic response was observed in $51.2 \%$ (21/41) with Lim-EE compared with $41.2 \%(63 / 153)$ with the Dif-EE $(P=0.29)$. Our data suggest that our reported clinicopathologic improvement is primarily driven by clinical improvement. Histopathologic remission is often harder to achieve and requires a longer period of follow-up, which may also explain the observed results. Most of our physicians recommend a follow-up EGD a few months following therapy for patients who have an elevated esophageal 
- Table3 Treatment at the time of initial and repeat esophagogastroduodenoscopy.

\begin{tabular}{|c|c|c|c|c|c|c|c|}
\hline & \multicolumn{2}{|l|}{ Initial EGD } & \multirow[t]{2}{*}{$P$ value } & & \multicolumn{2}{|c|}{ Repeat/follow-up EGD } & \multirow[t]{2}{*}{$P$ value } \\
\hline & $\begin{array}{l}\text { Lim-EE }^{1} \\
(n=48)\end{array}$ & $\begin{array}{l}\text { Dif-EE } \\
(n=167)\end{array}$ & & & $\begin{array}{l}\text { Lim-EE }^{1} \\
(n=48)\end{array}$ & $\begin{array}{l}\text { Dif-EE } \\
(n=167)\end{array}$ & \\
\hline $\begin{array}{l}\text { Elimination diet before } \\
\text { initial EGD/biopsy, n (\%) }\end{array}$ & 0 & $11(6.6)$ & 0.13 & $\begin{array}{l}\text { Elimination diet after initial } \\
\text { EGD/biopsy, } n(\%)\end{array}$ & $3(6.3)$ & $21(12.6)$ & 0.30 \\
\hline $\begin{array}{l}\text { Dilation performed at } \\
\text { time of initial EGD, n (\%) }\end{array}$ & $15(31.3)$ & $41(24.6)$ & \multirow[t]{4}{*}{0.36} & $\begin{array}{l}\text { Dilation performed at follow- } \\
\text { up EGD, } \mathrm{n}(\%)\end{array}$ & $16(33.3)$ & $42(25.1)$ & \multirow[t]{4}{*}{0.27} \\
\hline - Savory & 5 & 12 & & - Savory & 2 & 15 & \\
\hline - Maloney & 2 & 7 & & - Maloney & 2 & 10 & \\
\hline . TTS & 8 & 22 & & - TTS & 12 & 17 & \\
\hline $\begin{array}{l}\text { PPI use before EGD/ } \\
\text { biopsy, } \mathrm{n}(\%)\end{array}$ & $18(37.5)$ & $58(34.7)$ & 0.41 & $\begin{array}{l}\text { PPI use after initial EGD/ } \\
\text { biopsy, } \mathrm{n}(\%)\end{array}$ & $39(81.3)$ & $128(76.7)$ & 0.56 \\
\hline - PPI BID dosing, n (\%) & $13(72.2)$ & $36(62.1)$ & 0.77 & - PPI BID dosing, n (\%) & $27(69.2)$ & $95(74.2)$ & 0.54 \\
\hline $\begin{array}{l}\text { PPI total dose, mean } \\
\text { (SD), mg }\end{array}$ & $55.2(22.6)$ & $50.1(23.3)$ & 0.34 & $\begin{array}{l}\text { PPI total dose, mean (SD), } \\
\text { mg }\end{array}$ & $56.9(20.4)$ & $56.6(22.9)$ & 0.71 \\
\hline \multicolumn{3}{|l|}{ PPI type, n } & \multirow[t]{7}{*}{0.60} & PPI type, n & & & \multirow[t]{7}{*}{0.31} \\
\hline - Omeprazole & 6 & 22 & & - Omeprazole & 19 & 68 & \\
\hline - Esomeprazole & 2 & 13 & & - Esomeprazole & 5 & 17 & \\
\hline - Pantoprazole & 5 & 10 & & - Pantoprazole & 12 & 28 & \\
\hline - Dexlansoprazole & 1 & 2 & & - Dexlansoprazole & 0 & 1 & \\
\hline - Lansoprazole & 3 & 10 & & - Lansoprazole & 2 & 14 & \\
\hline - Rabeprazole & 1 & 1 & & - Rabeprazole & 1 & 0 & \\
\hline $\begin{array}{l}\text { Steroid use before EGD/ } \\
\text { biopsy, n (\%) }\end{array}$ & $4(8.3)$ & $16(9.6)$ & \multirow[t]{4}{*}{$>0.99$} & $\begin{array}{l}\text { Steroid after initial EGD/ } \\
\text { biopsy, } \mathrm{n}(\%)\end{array}$ & $5(10.4)$ & $29(17.4)$ & \multirow[t]{4}{*}{$>0.99$} \\
\hline - Fluticasone & 0 & 1 & & - Fluticasone & 4 & 21 & \\
\hline - Budesonide & 4 & 9 & & - Budesonide & 1 & 8 & \\
\hline - Prednisone & 0 & 7 & & - Prednisone & 0 & 0 & \\
\hline
\end{tabular}

eosinophil count. However, some patients did not comply with our recommendation for a repeat EGD and others did not comply with the timing of a repeat EGD. This could lead to an inevitable selection bias and also explain the lack of statistical significance observed in the histologic outcomes. Patients who remain symptomatic are more likely to attend their repeat EGD compared with those who respond to treatment. Therefore, we may be selectively analyzing a refractory EoE group in our histologic analysis. However, this bias should resolve by analyzing the clinical response as shown in our clinical and clinicopathologic outcomes analyses and results. It is also important to note that the analyzed response to treatment is a combined analysis of available treatments shown in > Table 3 , including elimination diet, PPI, and steroids. Given that the majority of patients were treated with PPIs, the observed response to treatment is likely related to PPI therapy. Unfortunately, we were not able to perform subanalyses based on treatment methods be- cause of the small number of patients in each category, which prevents statistical significance from being determined. With regard to the change in treatments between the initial and follow-up EGD, this is likely explained by the treatment protocol we use for EoE. Our protocol recommends that after a diagnosis of EoE is made, the first step should be PPI therapy and possibly consideration for elimination diet. If the patient is taking oncedaily PPI, the next step is to consider up-titrating to twice daily. Steroids are usually utilized in refractory cases. Therefore, more patients were on PPIs and twice-daily dosing by the time they underwent repeat endoscopies compared with the initial EGD. At the time of follow-up EGD, we would expect the number of patients prescribed steroids to be higher if they were refractory to twice-daily PPIs. In addition, it remains uncertain whether the EoE response to PPI is due to its anti-inflammatory mechanisms, such as decreasing eotaxin-3 expression, or its anti-secretory activity from coexistent GERD [23]. A recent study by 
Sawada et al. focused on the extent of eosinophilia and PPI responsiveness. The authors reported that the prevalence of PPI responders was significantly higher in the limited group compared with the diffuse group ( $100 \%$ vs. $63 \%$; $P=0.01)$, which supports our hypothesis [24]. It is important to note that both the Sawada et al. study and the current study are similar, but not identical. The definition of disease extent was different; Sawada et al. defined limited disease as an endoscopic lesion confined to one-third of the esophagus (upper, middle, or lower), whereas in the current study Lim-EE was defined as distal disease. In addition, Sawada et al. defined Lim-EE and Dif-EE endoscopically whereas our study defined EoE histologically.

Our study is not free of limitations. First, we grouped patients based on the extent of eosinophilia seen on the initial EGD, but some patients had already been treated with a PPI before being seen at our referral center, which may have led to mosaic grouping. In order to address this, we performed a subanalysis of the clinicopathologic response after excluding patients who were on PPI or steroids at the time of biopsy during their initial EGD. A total of $28 / 48$ patients (58.3\%) with Lim-EE and $68 / 156$ (43.6\%) with Dif-EE had clinicopathologic response to treatment $(P=0.06)$. This borderline significance is likely reflective of the inadequate sample size and power loss after excluding $40 \%$ of the original cohort. This trend would likely be more significant with a larger cohort. Second, the majority of our patients did not undergo $\mathrm{pH}$ monitoring studies, which limited our ability to distinguish between GERD-esophagitis and EoE-esophagitis; therefore, we defined GERD on the basis of its known clinical features. Third, our sample size did not allow us to substratify patients or assess the outcomes based on different treatment regimens. We were also unable to track treatment changes during the follow-up period that could have influenced the observed outcomes. In addition, our evaluation of clinical responses was based on physicians' documentation in the medical records rather than a validated questionnaire. Finally, the limited follow-up and the retrospective nature of the study may have introduced other potential biases.

Despite these limitations, we believe that our study provides valuable information that addresses a gap in the scientific literature and which also adds value to clinical practice. We propose an added benefit of separating proximal and distal samples into different jars to determine the extent of disease. Although the use of separate jars increases overall cost, the information obtained provides valuable clinical information with regard to differences in expected response to various treatments. Prior studies have postulated a potential overlap between GERD and EoE, and our study now provides clinical data in support of this concept [6]. Therefore, in symptomatic patients with limited EoE who fail to respond to conventional PPI therapy, upscaling of treatment following the algorithm of EoE should be considered. Prospective studies are needed to confirm our observations, and in particular to address the potential correlation between the location of typical endoscopic findings of EoE and the histology-based EoE phenotype (limited vs. diffuse). It may also be of interest to investigate the clinical significance of esophageal eosinophilia limited to the proximal part of the esophagus.
In conclusion, our study suggests that biopsies from multiple esophageal locations may help stratify treatment outcomes in patients with EoE. Patients with EoE limited to the distal esophagus responded better to PPI therapy than those with diffuse esophageal involvement. This may represent a population of patients with GERD or milder EoE disease. Those with diffuse involvement may represent a group less likely to respond to therapy.

\section{Competing interests}

The authors declare that they have no conflict of interest.

\section{References}

[1] Dellon ES, Hirano I. Epidemiology and natural history of eosinophilic esophagitis. Gastroenterology 2018; 154: 319-332

[2] Dellon ES. Approach to diagnosis of eosinophilic esophagitis. Gastroenterol Hepatol (N Y) 2011; 7: 742-744

[3] Dellon ES, Gonsalves N, Hirano I et al. ACG Clinical Guideline: Evidenced based approach to the diagnosis and management of esophageal eosinophilia and eosinophilic esophagitis (EoE). Am J Gastroenterol 2013; 108: 679-692

[4] Lucendo AJ, Molina-Infante J, Arias A et al. Guidelines on eosinophilic esophagitis: evidence-based statements and recommendations for diagnosis and management in children and adults. United European Gastroenterol J 2017; 5: 335-358

[5] Gonsalves N, Policarpio-Nicolas M, Zhang Q et al. Histopathologic variability and endoscopic correlates in adults with eosinophilic esophagitis. Gastrointest Endosc 2006; 64: 313-319

[6] Spechler S]. Gastroesophageal reflux disease and eosinophilic esophagitis. Gastroenterol Hepatol (N Y) 2019; 15: 111-113

[7] Lee $S$, de Boer WB, Naran A et al. More than just counting eosinophils: proximal oesophageal involvement and subepithelial sclerosis are major diagnostic criteria for eosinophilic oesophagitis. J Clin Pathol 2010; 63: 644-647

[8] Alkhattabi M, Kubica M, Driman D et al. A225 The utility of separate distal and mid-esophageal biopsies in the diagnosis of eosinophilic esophagitis (EoE). J Can Assoc Gastroenterol 2018; 1: 392-393

[9] Molina-Infante J, Ferrando-Lamana L, Ripoll C et al. Esophageal eosinophilic infiltration responds to proton pump inhibition in most adults. Clin Gastroenterol Hepatol 2011; 9: 110-117

[10] Liacouras CA, Furuta GT, Hirano I et al. Eosinophilic esophagitis: updated consensus recommendations for children and adults. J Allergy Clin Immunol 2011; 128: 3-20

[11] Saffari H, Peterson KA, Fang JC et al. Patchy eosinophil distributions in an esophagectomy specimen from a patient with eosinophilic esophagitis: implications for endoscopic biopsy. J Allergy Clin Immunol 2012; 130: 798-800

[12] Dellon ES, Liacouras CA, Molina-Infante J et al. Updated international consensus diagnostic criteria for eosinophilic esophagitis: proceedings of the AGREE conference. Gastroenterology 2018; 155: 10221033

[13] Frazzoni M, Penagini R, Frazzoni L et al. Role of reflux in the pathogenesis of eosinophilic esophagitis: comprehensive appraisal with offand on PPI impedance-pH monitoring. Am J Gastroenterol 2019; 114 : 1606-1613 
[14] Winter HS, Madara JL, Stafford RJ et al. Intraepithelial eosinophils: a new diagnostic criterion for reflux esophagitis. Gastroenterology 1982; 83: 818-823

[15] Rodrigo S, Abboud G, Oh D et al. High intraepithelial eosinophil counts in esophageal squamous epithelium are not specific for eosinophilic esophagitis in adults. Am J Gastroenterol 2008; 103: 435442

[16] Sayej WN, Patel R, Baker RD et al. Treatment with high-dose proton pump inhibitors helps distinguish eosinophilic esophagitis from noneosinophilic esophagitis. J Pediatr Gastroenterol Nutr 2009; 49: 393-399

[17] Spechler S], Genta RM, Souza RF. Thoughts on the complex relationship between gastroesophageal reflux disease and eosinophilic esophagitis. Am J Gastroenterol 2007; 102: 1301-1306

[18] Shah NA, Albert DM, Hall NM et al. Managing eosinophilic esophagitis: challenges and solutions. Clin Exp Gastroenterol 2016; 9: 281290

[19] Gomez-Aldana A, Jaramillo-Santos M, Delgado A et al. Eosinophilic esophagitis: current concepts in diagnosis and treatment. World ] Gastroenterol 2019; 25: 4598-4613
[20] Lucendo A], Arias A, Molina-Infante J. Efficacy of proton pump inhibitor drugs for inducing clinical and histologic remission in patients with symptomatic esophageal eosinophilia: a systematic review and meta-analysis. Clin Gastroenterol Hepatol 2016; 14: 13-22

[21] Gomez-Torrijos E, Garcia-Rodriguez R, Castro-jimenez A et al. The efficacy of step-down therapy in adult patients with proton pump inhibitor-responsive oesophageal eosinophilia. Aliment Pharmacol Ther 2016; 43: 534-540

[22] Lucendo A], Miehlke S, Schlag C et al. Efficacy of budesonide orodispersible tablets as induction therapy for eosinophilic esophagitis in a randomized placebo-controlled trial. Gastroenterology 2019; 157: $74-86$

[23] Eluri S, Dellon ES. Proton pump inhibitor-responsive oesophageal eosinophilia and eosinophilic oesophagitis: more similarities than differences. Curr Opin Gastroenterol 2015; 31: 309-315

[24] Sawada A, Hashimoto A, Uemura R et al. Association between endoscopic findings of eosinophilic esophagitis and responsiveness to proton pump inhibitors. Endosc Int Open 2019; 7: E433-E439 